Homology, Homotopy and Applications, vol.21(2), 2019, pp.1-22

\title{
POSET-STRATIFIED SPACE STRUCTURES OF HOMOTOPY SETS
}

\author{
TOSHIHIRO YAMAGUCHI AND SHOJI YOKURA
}

(communicated by Jonathan M. Rosenberg)

\begin{abstract}
A poset-stratified space is a pair $(S, S \stackrel{\pi}{\rightarrow} P)$ of a topological space $S$ and a continuous map $\pi: S \rightarrow P$ with a poset $P$ considered as a topological space with its associated Alexandroff topology. In this paper we show that one can impose such a posetstratified space structure on the homotopy set $[X, Y]$ of homotopy classes of continuous maps by considering a canonical but nontrivial order (preorder) on it, namely we can capture the homotopy set $[X, Y]$ as an object of the category of poset-stratified spaces. The order we consider is related to the notion of dependence of maps (by Karol Borsuk). Furthermore via homology and cohomology the homotopy set $[X, Y]$ can have other poset-stratified space structures. In the cohomology case, we get some results which are equivalent to the notion of dependence of cohomology classes (by René Thom) and we can show that the set of isomorphism classes of complex vector bundles can be captured as a poset-stratified space via the poset of the subrings consisting of all the characteristic classes. We also show that some invariants such as Gottlieb groups and Lusternik-Schnirelmann category of a map give posetstratified space structures to the homotopy set $[X, Y]$.
\end{abstract}

\section{Introduction}

The homotopy set $[X, Y]$ is the set of homotopy classes of continuous maps from a topological space $X$ to another one $Y$. In our previous work [40] we consider a preorder on the homotopy set $[X, Y]$ using the action of the self-homotopy equivalences $\mathcal{E}[X]$ of $X$ and the self-homotopy equivalences $\mathcal{E}[Y]$ of $Y$ on $[X, Y]$. Using such a preordered set (proset), we consider some classification of Hurewicz fibrations.

In this paper we consider another preorder on $[X, Y]$ via the action of monoids $[X, X]$ and $[Y, Y]$ on $[X, Y]$, instead of $\mathcal{E}[X]$ and $\mathcal{E}[Y]$. Here we note that a homotopy class $[f] \in \mathcal{E}[X]$ has its inverse $[f]^{-1} \in \mathcal{E}[X]$, but a homotopy class $[f] \in[X, X]$ does not always have an inverse $[f]^{-1} \in[X, X]$, which is a substantial difference between

S. Yokura is partially supported by JSPS KAKENHI Grant Number 16H03936.

Received October 27, 2017, revised September 13, 2018; published on December 19, 2018.

2010 Mathematics Subject Classification: 06A06, 18A99, 54B99, 55P20, 55P10, 55P62, 55P99.

Key words and phrases: homotopy set, poset, poset-stratified space, Alexandroff topology, dependence of maps, dependence of cohomology classes, Sullivan minimal model.

Article available at http://dx.doi.org/10.4310/HHA.2019.v21.n2.a1

Copyright (C) 2018, International Press. Permission to copy for private use granted. 
$[X, X]$ and $\mathcal{E}[X]$. For example, we consider the following order:

$$
[f] \varliminf_{R}[g] \Longleftrightarrow \exists[s] \in[X, X] \text { such that }[f]=[g] \circ[s],
$$

i.e., the following diagram commutes up to homotopy $(f \sim g \circ s)$ :

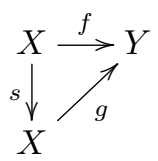

This is a preorder. Then we consider the following equivalence relation $\sim_{R}$ using this preorder $\leqq_{R}$ :

$$
[f] \sim_{R}[g] \Longleftrightarrow[f] \leqq_{R}[g] \text { and }[g] \leqq_{R}[f]
$$

namely,

$$
\exists\left[s_{1}\right],\left[s_{2}\right] \in[X, X] \text { such that }[f]=[g] \circ\left[s_{1}\right],[g]=[f] \circ\left[s_{2}\right],
$$

i.e., the following diagram commutes up to homotopy:

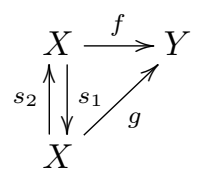

The relation $\sim_{R}$ is an equivalence relation, called right equivalence relation and the set of equivalence classes shall be denoted by $[X, Y]_{R}:=[X, Y] / \sim_{R}$. The equivalence class of $[f]$ is denoted by $[f]_{R}$. We define the order $\varliminf_{R}^{\prime}$ on $[X, Y]_{R}$ by

$$
[f]_{R} \leqq_{R}^{\prime}[g]_{R} \Longleftrightarrow[f] \leqq_{R}[g] .
$$

This order $\leqq_{R}^{\prime}$ is well-defined and becomes a partial order. Thus the canonical map $\pi_{R}:\left([X, Y], \leqq_{R}\right) \rightarrow\left([X, Y]_{R}, \leqq_{R}^{\prime}\right)$ is a monotone (order-preserving) map from a proset to a poset. If we consider the Alexandroff topologies $\tau_{\varliminf_{R}}$ on the source $\left([X, Y], \leqq_{R}\right)$ and $\tau_{\varliminf_{R}^{\prime}}$ on the target $\left([X, Y]_{R}, \leqq_{R}^{\prime}\right)$, this in turn gives us a continuous map $\pi_{R}:\left([X, Y], \tau_{\varliminf_{R}}\right) \rightarrow\left([X, Y]_{R}, \tau_{\varliminf_{R}^{\prime}}\right)$. In other words, this is a continuous map from a topological space $\left([X, Y], \tau_{\varliminf_{R}}\right)$ to a poset $\left([X, Y]_{R}, \varliminf_{R}^{\prime}\right)$ which is considered as a topological space $\left([X, Y]_{R}, \tau_{\varliminf_{R}^{\prime}}\right)$ with the Alexandroff topology. Such a map is called $a$ poset-stratified space in modern terminology (e.g., see [23]).

Remark 1.1. In the case when we consider the self-homotopy equivalences $\mathcal{E}[X]$ of $X$, instead of the monoid $[X, X]$, since each element $[s] \in \mathcal{E}[X]$ has its inverse $[s]^{-1} \in \mathcal{E}[X]$ (more precisely, $\exists s^{\prime}: X \rightarrow X$ such that $s \circ s^{\prime} \sim i d_{X}$ and $s^{\prime} \circ s \sim i d_{X}$, thus $[s]^{-1}=\left[s^{\prime}\right]$ ), the above equivalence relation $\sim_{R}$ is replaced simply by the following equivalence relation $\sim_{\mathcal{E} R}$ :

$$
[f] \sim_{\mathcal{E} R}[g] \Longleftrightarrow \exists[s] \in \mathcal{E}[X] \text { such that }[f]=[g] \circ[s],
$$

i.e., the following diagram commutes up to homotopy $(f \sim g \circ s)$ :

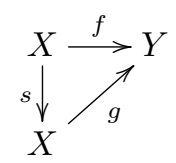


Because $f \sim g \circ s$ automatically implies that $g \sim f \circ s^{\prime}$. On the set $[X, Y]_{\mathcal{E} R}$ of equivalence classes, as in the case of $[X, Y]_{R}$, one can define the following order for $[f]_{\mathcal{E} R},[g]_{\mathcal{E} R} \in[X, Y]_{\mathcal{E} R}$

$$
[f]_{\mathcal{E} R} \leqq_{R}[g]_{\mathcal{E} R} \Longleftrightarrow \exists s \in[X, X](\text { not } \exists s \in \mathcal{E}[X]) \text { such that }[f]=[g] \circ[s] .
$$

Here we emphasize that this order is not necessarily a partial order, but that the above order $\varliminf_{R}^{\prime}$ on $[X, Y]_{R}$ defined by $[f]_{R} \leqq_{R}^{\prime}[g]_{R} \Longleftrightarrow[f] \leqq_{R}[g]$ is a partial order, because of the equivalence relation $[f] \sim_{R}[g]$ defined by $\exists\left[s_{1}\right],\left[s_{2}\right] \in[X, X]$ such that $[f]=[g] \circ\left[s_{1}\right],[g]=[f] \circ\left[s_{2}\right]$. One could think of such a pair $\left(\left[s_{1}\right],\left[s_{2}\right]\right)$ as a "mock" self-homotopy equivalence of $X$ with respect to the pair $(f, g)$.

Similarly we consider the preorder

$$
[f] \leqq_{L}[g] \Longleftrightarrow \exists[t] \in[Y, Y] \text { such that }[f]=[t] \circ[g],
$$

i.e., the following diagram commutes up to homotopy $\left(f \sim t \circ g^{1}\right)$ :

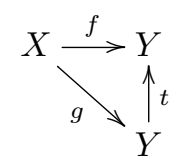

Then we consider the following equivalence relation $\sim_{L}$ using this preorder $\leqq_{L}$ :

$$
[f] \sim_{L}[g] \Longleftrightarrow[f] \leqq_{L}[g] \text { and }[g] \leqq_{L}[f],
$$

i.e., $\exists\left[t_{1}\right],\left[t_{2}\right] \in[Y, Y]$ such that $[f]=\left[t_{1}\right] \circ[g],[g]=\left[t_{2}\right] \circ[f]$, i.e., the following diagram commutes up to homotopy:

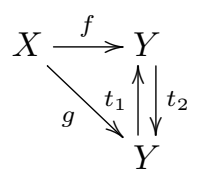

The equivalence relation $\sim_{L}$ is called left equivalence relation and the set of equivalence classes shall be denoted $[X, Y]_{L}:=[X, Y] / \sim_{L}$. As in the case of $\leqq_{R}$ and $\leqq_{R}^{\prime}$, the canonical map $\pi_{L}:\left([X, Y], \leqq_{L}\right) \rightarrow\left([X, Y]_{L}, \leqq_{L}^{\prime}\right)$ is a monotone map from a proset to a poset.

These poset-stratified spaces can be captured as functors from the homotopy category of topological spaces to the category of poset-stratified spaces as follows:

Theorem 1.2. Let $h \mathcal{T}$ op be the homotopy category of topological spaces.

1. For any object $S \in O b j(h \mathcal{T}$ op), we have an associated covariant functor $\mathfrak{s t}_{*}^{S}: h \mathcal{T}$ op $\rightarrow$ Strat such that

(a) for each object $Y \in \operatorname{Obj}(h \mathcal{T} o p)$,

$$
\mathfrak{s t}_{*}^{S}(X):=\left(\left([S, X], \tau_{\varliminf_{R}}\right),\left([S, X], \tau_{\varliminf_{R}}\right) \stackrel{\pi_{R}}{\longrightarrow}\left([S, X]_{R}, \leqq_{R}^{\prime}\right)\right)
$$

\footnotetext{
${ }^{1}$ As remarked later, in a different context Borsuk $[6,7]$ considered such a relation when he characterized his definition of $f: X \rightarrow Y$ depending on $g: X \rightarrow Y$.
} 
(b) for a morphism $[f] \in[X, Y], \mathfrak{s t}_{*}^{S}([f])$ is the following commutative diagram:

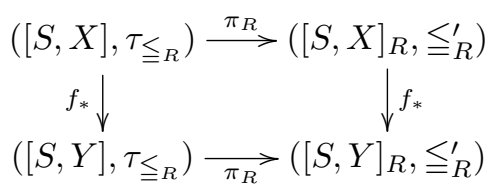

2. For any object $T \in O b j(h \mathcal{T} o p)$, we have an associated contravariant functor $\mathfrak{s t}_{T}^{*}: h \mathcal{T}$ op $\rightarrow$ Strat such that

(a) for each object $X \in \operatorname{Obj}(h \mathcal{T}$ op $)$,

$$
\mathfrak{s t}_{T}^{*}(X):=\left(\left([X, T], \tau_{\leqq_{L}}\right),\left([X, T], \tau_{\leqq_{L}}\right) \stackrel{\pi_{L}}{\longrightarrow}\left([X, T]_{L}, \leqq_{L}^{\prime}\right)\right)
$$

(b) for a morphism $[f] \in[X, Y], \mathfrak{s t}_{T}^{*}([f])$ is the following commutative diagram:

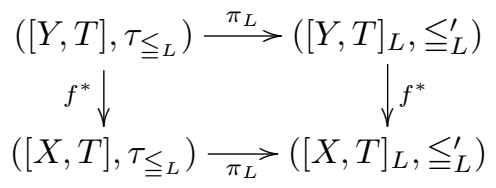

In Example 3.7 we see an example where the homotopy sets are the same: $[S, X]=$ $[S, Y]$, but their poset-stratified space structures are different: $\mathfrak{s t}_{*}^{S}(X) \neq \mathfrak{s t}_{*}^{S}(Y)$.

By considering homology and cohomology, and homotopy and cohomotopy, we can get other more "algebraic" or "geometric" poset-stratified space structures on the homotopy set. For example, consider the homotopy set $\left[S^{1}, S^{1}\right]=\mathbb{Z}$. Then the preorder $a \leqq_{R} b$ is by our definition nothing but $\exists s \in \mathbb{Z}$ such that $a=b \cdot s$, i.e., $b$ divides $a, b \mid a$. For an integer $n \in \mathbb{Z}=\left[S^{1}, S^{1}\right]$, i.e., $n$ is the homotopy class of the map $z^{n}: S^{1} \rightarrow S^{1}$ and consider $\left(z^{n}\right)_{*}: H_{1}\left(S^{1}\right) \rightarrow H_{1}\left(S^{1}\right)$ or $\left(z^{n}\right)_{*}: \pi_{1}\left(S^{1}\right) \rightarrow \pi_{1}\left(S^{1}\right)$, which gives us the homomorphism $\times n: \mathbb{Z} \rightarrow \mathbb{Z}$. Then the image $\operatorname{Im}(\times n)=(n)=$ $\{k n \mid k \in \mathbb{Z}\}$ is the subgroup generated by the integer $n$. The set $\mathcal{S} u b(\mathbb{Z})$ of all the subgroups of $\mathbb{Z}$ is $\{(n) \mid n \in \mathbb{Z}\}$ and the order $(a) \leqq(b)$ defined by the inclusion (a) $\subset(b)$, which means that $\exists s \in \mathbb{Z}$ such that $a=b \cdot s$, thus $b \mid a$. Thus the map $\operatorname{Im}_{H_{1}}:\left(\left[S^{1}, S^{1}\right], \leqq_{R}\right)=\left(\mathbb{Z}, \leqq_{R}\right) \rightarrow(\mathcal{S} u b(\mathbb{Z}), \leqq)$ defined by $\operatorname{Im}_{H_{1}}(n)=\operatorname{Im}\left(\left(z^{n}\right)_{*}\right)=$ $(n)$ is a monotone map.

In the case of $\left([X, Y], \leqq_{L}\right)$ we consider the cohomology theory $H^{*}(-; \mathbb{Z})$ and we get a canonical monotone map $\operatorname{Im}_{H^{*}}:\left([X, Y], \leqq_{L}\right) \rightarrow\left(\mathcal{S} u b\left(H^{*}(X)\right), \leqq\right)$, which is defined by $\operatorname{Im}_{H^{*}}([f]):=\operatorname{Im}\left(f^{*}: H^{*}(Y) \rightarrow H^{*}(X)\right)=f^{*}\left(H^{*}(Y)\right)$. Here $\mathcal{S} u b\left(H^{*}(X)\right)$ is the set of all the subgroups of $H^{*}(X)$ and the order $S_{1} \leqq S_{2}$ for subgroups $S_{1}, S_{2} \in$ $\mathcal{S} u b\left(H^{*}(X)\right)$ is the usual inclusion $S_{1} \subset S_{2}$. This monotone map

$$
\operatorname{Im}_{H^{*}}:\left([X, Y], \leqq_{L}\right) \rightarrow\left(\mathcal{S} u b\left(H^{*}(X)\right), \leqq\right)
$$

has a connection with Thom's notion of dependence of cohomology classes [36]. Indeed, let us consider $Y=K(\mathbb{Z}, p)$ the Eilenberg-Maclane space, then we have

$$
\operatorname{Im}_{H^{*}}:([X, K(\mathbb{Z}, p)], \leqq L) \rightarrow\left(\mathcal{S} u b\left(H^{*}(X)\right), \leqq\right) .
$$

Since $H^{p}(X, \mathbb{Z})=[X, K(\mathbb{Z}, p)]$, let $f_{\alpha}: X \rightarrow K(\mathbb{Z}, p)$ be a map whose homotopy class $\left[f_{\alpha}\right]$ corresponds to the cohomology class $\alpha \in H^{p}(X, \mathbb{Z})$. Let $\beta \in H^{p}(X, \mathbb{Z})$ be another cohomology class, thus we consider the corresponding homotopy class $\left[f_{\beta}\right]$. Let $\left[f_{\beta}\right] \leqq_{L}\left[f_{\alpha}\right]$, i.e., $\exists[t] \in[K(\mathbb{Z}, p), K(\mathbb{Z}, p)]$ such that $\left[f_{\beta}\right]=[t] \circ\left[f_{\alpha}\right]\left(f_{\beta} \sim t \circ f_{\alpha}\right)$, which 
implies that

$$
\operatorname{Im}\left(f_{\beta}^{*}\right)=f_{\beta}^{*}\left(H^{*}(K(\mathbb{Z}, p))\right) \subset \operatorname{Im}\left(f_{\alpha}^{*}\right)=f_{\alpha}^{*}\left(H^{*}(K(\mathbb{Z}, p))\right) .
$$

In particular, $\beta \in f_{\beta}^{*}\left(H^{*}(K(\mathbb{Z}, p))\right)$, thus $\beta \in f_{\alpha}^{*}\left(H^{*}(K(\mathbb{Z}, p))\right)$, which implies by Thom's definition of dependence of cohomology classes [36] (also see [18]) that the cohomology class $\beta$ depends on the cohomology class $\alpha$. Thus the upshot is that our $\left[f_{\beta}\right] \leqq_{L}\left[f_{\alpha}\right]$, namely, that $f_{\beta}$ depends on $f_{\alpha}$ (using Borsuk's definition of dependence of maps) implies that $\beta$ depends on $\alpha$.

If we consider $Y=G_{n}\left(\mathbb{C}^{\infty}\right)$ the infinite Grassmann of $n$-dimensional planes in $\mathbb{C}^{\infty}$ for $\operatorname{Im}_{H^{*}}:\left([X, Y], \leqq_{L}\right) \rightarrow\left(\mathcal{S} u b\left(H^{*}(X)\right), \leqq\right)$, then we get a natural "order" among the isomorphism classes of complex vector bundles. Indeed, if we denote the set of isomorphism classes of complex vector bundles of rank $n$, then we know that $\operatorname{Vect}_{n}(X) \cong$ $\left[X, G_{n}\left(\mathbb{C}^{\infty}\right)\right]$, which is by the correspondence $[E] \leftrightarrow\left[f_{E}\right]$, where $f_{E}: X \rightarrow G_{n}\left(\mathbb{C}^{\infty}\right)$ is a classifying map of $E$, i.e., $E=f_{E}^{*} \gamma^{n}$, where $\gamma^{n}$ is the universal complex vector bundle of rank $n$ over $G_{n}\left(\mathbb{C}^{\infty}\right)$. By the isomorphism $\operatorname{Vect}_{n}(X) \cong\left[X, G_{n}\left(\mathbb{C}^{\infty}\right)\right]$ we can consider the preorder on $\operatorname{Vect}_{n}(X):[E] \leqq_{L}[F] \Longleftrightarrow\left[f_{E}\right] \leqq_{L}\left[f_{F}\right]$, where $f_{E}, f_{F}: X \rightarrow$ $G_{n}\left(\mathbb{C}^{\infty}\right)$ are respectively the classifying maps of $E$ and $F$. Then we have the following well-defined monotone (order-preserving) map:

$$
\operatorname{Im}_{H^{*}}:\left(\operatorname{Vect}_{n}(X), \leqq_{L}\right) \rightarrow\left(\mathcal{S} u b\left(H^{*}(X ; \mathbb{Z})\right), \leqq\right)
$$

defined by $\operatorname{Im}_{H^{*}}([E]):=\operatorname{Im}\left(f_{E}^{*}: H^{*}\left(G_{n}\left(\mathbb{C}^{\infty}\right) ; \mathbb{Z}\right) \rightarrow H^{*}(X ; \mathbb{Z})\right)$. By the definition of characteristic classes, $\operatorname{Im}\left(f_{E}^{*}: H^{*}\left(G_{n}\left(\mathbb{C}^{\infty}\right) ; \mathbb{Z}\right) \rightarrow H^{*}(X ; \mathbb{Z})\right)$ is the subring consisting of all the characteristic classes of $E$, denoted by $\operatorname{Char}(E)$. Therefore we have $[E] \leqq_{L}$ $[F] \Longrightarrow \operatorname{Char}(E) \subseteq \operatorname{Char}(F)$. We also get that $[E] \sim_{L}[F] \Longrightarrow \operatorname{Char}(E)=\operatorname{Char}(F)$.

We also show that the Gottlieb groups and Lusternik-Schnirelmann category of a map give poset-stratified space structures to homotopy sets.

\section{Preliminaries}

In this section we give some preliminaries for later use.

A preorder on a set $P$ is a relation $\leqq$ which is reflexive $(a \leqq a)$ and transitive $(a \leqq b, b \leqq c \Longrightarrow a \leqq c)$. A set $(P, \leqq)$ equipped with a preorder $\leqq$ is called a proset (preordered set). If a preorder $\leqq$ is anti-symmetric $(a \leqq b, b \leqq a \Longrightarrow a=b)$, then it is called a partial order and a set with a partial order is called a poset (partially ordered set).

Definition 2.1 (Alexandroff topology [1]). Let $X$ be a topological space. If the intersection of any family of open sets is open or equivalently the union of any family of closed sets is closed, then the topology is called an Alexandroff topology and the space is called an Alexandroff space.

For Alexandroff topology or spaces, e.g., see [1, 2, 3], [9, §4.2.1 Alexandroff Topology], [33], [39, Appendix A Pre-orders and spaces].

Note that any finite topological space, i.e., a finite set with a topology, is clearly an Alexandroff space. (For finite topological spaces, e.g., see [5, 25, 26, 27, 29, 30, 34].)

Given a proset $(X, \leqq)$, we define $U \subset X$ to be an open set if and only if $x \in U$, 
$x \leqq y \Longrightarrow y \in U$, i.e., if and only if $U$ is closed upwards ${ }^{2}$. In other words, if we let $U_{x}:=\{y \in X \mid x \leqq y\}$, then $\left\{U_{x} \mid x \in X\right\}$ is the base for the topology. This topology is denoted by $\tau_{\leqq}$.

Lemma 2.2. For a proset $(X, \leqq)$, the topological space $\left(X, \tau_{\leqq}\right)$is an Alexandroff space.

Because of this, the topology $\tau_{\leqq}$is called the Alexandroff topology (associated to the preorder).

Observation 2.3. A subset $F$ is a closed set in the topology $\tau_{\leqq}$if and only if $x \in F, y \leqq x \Longrightarrow y \in F$.

From this observation we can see that if $P$ is a poset, not a proset, for any point $x \in P,\{x\}=\{y \in P \mid x \leqq y\} \cap\{y \in P \mid y \leqq x\}$. In other words, in the associated Alexandroff topology $\tau_{\leq}$any singleton $\{x\}$ is a locally closed set, i.e., the intersection of a closed set and an open set. Note that for example, for a two-point proset $(\{a, b\}, \leqq)$ with the preorder $\leqq$ defined by $a \leqq b, b \leqq a$, the above observation does not hold.

If we let $\mathcal{P}$ roset be the category of prosets and monotone (order-preserving) functions of prosets and $\mathcal{A l e x}$ be the category of Alexandroff spaces and continuous maps, then we have a covariant functor $\mathcal{T}: \mathcal{P}$ roset $\rightarrow$ Alex.

Conversely, for a topological space $(X, \tau)$, we define the following order, called specialization order, on $X: x \leqq_{\tau} y \Longleftrightarrow x \in \overline{\{y\}}$. Certainly this is a preorder, but not necessarily a partial order. (For example, for any indiscrete topological space having more than or equal to two points, it is never a partial order.) If $f:\left(X, \tau_{1}\right) \rightarrow$ $\left(Y, \tau_{2}\right)$ is a continuous map, then $f:\left(X, \leqq_{\tau_{1}}\right) \rightarrow\left(Y, \leqq_{\tau_{2}}\right)$ is a monotone function. Therefore we have a covariant functor $\mathcal{P}: \mathcal{T}$ op $\rightarrow$ Proset. We have that for any proset $(X, \leqq),(\mathcal{P} \circ \mathcal{T})((X, \leqq))=(X, \leqq)$, i.e., $\mathcal{P} \circ \mathcal{T}=I d_{\mathcal{P} \text { roset }}$. However, in general, for a topological space $(X, \tau)$ we have $(\mathcal{T} \circ \mathcal{P})((X, \tau)) \neq(X, \tau)$, i.e., $\mathcal{T} \circ \mathcal{P} \neq$ $I d_{\mathcal{T} \text { op }}$. The reason is simple: $(\mathcal{T} \circ \mathcal{P})((X, \tau))$ is always an Alexandroff space, even if the original space $(X, \tau)$ is not an Alexandroff space, namely the topology of $(\mathcal{T} \circ \mathcal{P})((X, \tau))$ is stronger that the original topology $\tau$. However, if we restrict the covariant functor $\mathcal{P}: \mathcal{T}$ op $\rightarrow \mathcal{P}$ roset to the subcategory $\mathcal{A l e x}$ of Alexandroff spaces, then we have $(\mathcal{T} \circ \mathcal{P})((X, \tau))=(X, \tau)$, i.e., $\mathcal{T} \circ \mathcal{P}=I d_{\mathcal{A l e x}}$. Therefore we have that $\mathcal{P} \circ \mathcal{T}=I d_{\mathcal{P r o s e t}}, \mathcal{T} \circ \mathcal{P}=I d_{\mathcal{A l e x}}$. Thus Alexandroff spaces and prosets are equivalent.

For a proset $(P, \leqq)$, we can consider the reversed order, denoted $\leqq o p$, by $a \leqq o p$ $b \Longleftrightarrow b \leqq a$. Here we note that the Alexandroff topologies associated to the two prosets $(P, \leqq)$ and $(P, \leqq o p)$ of the same set $P$ are different.

A stratification of a topological space (which can be the underlying topological space of a much finer object such as a complex algebraic variety, a complex analytic space) is a special kind of decomposition with certain extra conditions. It seems that there is no fixed or standard definition of stratification and there are several ones

\footnotetext{
${ }^{2}$ The Alexandroff topology is sometimes considered by defining an open set to be closed downwards instead of closed upwards, e.g., see [3, 5, 25, 33]. When stratification theory or poset-stratified spaces are considered as in the above cited references [9] and [39], upward closedness is used in defining Alexandroff topology (e.g., see [23, Definition A.5.1] and [35, Definition 2.1 ] as well).
} 
depending on the objects to study, such as topologically stratified spaces and ThomWhitney stratified spaces. In [35] Tamaki gives a nice review of several stratifications available in mathematics.

Here is one definition of stratification:

Definition 2.4. Let $X$ be a topological space. If a family $\left\{e_{\lambda}\right\}_{\lambda \in \Lambda}$ of subsets of $X$ satisfies the following conditions, then $\left\{e_{\lambda}\right\}_{\lambda \in \Lambda}$ is called a stratification of $X$ :

1. $e_{\lambda} \cap e_{\mu}=\emptyset$ if $\lambda \neq \mu$.

2. $X=\bigcup_{\lambda} e_{\lambda}$.

3. (locally closed set) Each $e_{\lambda}$ is a locally closed set.

4. (frontier condition) $e_{\lambda} \cap \overline{e_{\mu}} \neq \emptyset \Longrightarrow e_{\lambda} \subset \overline{e_{\mu}}$.

Just a decomposition requires only (1) and (2). Given a decomposition $\mathcal{D}$ of $X$, we have the quotient map $\pi_{\mathcal{D}}: X \rightarrow X / \mathcal{D}$, which means that one considers each piece $e_{\lambda}$ as a point. Then we can identify $X / \mathcal{D}=\Lambda$. We consider the quotient topology, denoted $\tau_{\pi_{\mathcal{D}}}$, on the target $\Lambda$, i.e., the finest or strongest topology on $\Lambda$ such that the quotient map $\pi_{\mathcal{D}}: X \rightarrow X / \mathcal{D}=\Lambda$ becomes a continuous map. Suppose that the quotient topology $\tau_{\pi_{\mathcal{D}}}$ is an Alexandroff topology, which is the case when the decomposition $\mathcal{D}$ is finite, i.e., $\Lambda$ is a finite set. Then we get the preorder $\leqq_{\tau_{\pi_{\mathcal{D}}}}$. If $\leqq_{\tau_{\pi_{\mathcal{D}}}}$ is a partial order, then each piece $e_{\lambda}=\pi_{\mathcal{D}}^{-1}(\lambda)$ has to be locally closed, because each singleton $\{\lambda\}$ is a locally closed set as observed above. At the moment we do not know if the converse holds, i.e., whether each piece $e_{\lambda}$ being locally closed implies that $\leqq_{\tau_{\pi_{\mathcal{D}}}}$ is a partial order.

As to the preorder on $\Lambda$, we can define it using the above "frontier condition" by $\lambda \varliminf^{*} \mu \Longleftrightarrow e_{\lambda} \subset \overline{e_{\mu}}$. Then one can see that each piece $e_{\lambda}$ being locally closed implies that the above preorder $\leqq^{*}$ is, in fact, a partial order. Furthermore the quotient map $\pi_{\mathcal{D}}: X \rightarrow X / \mathcal{D}=\Lambda$ is a continuous map with the Alexandroff topology $\tau_{\leqq *}$ associated to the order $\leqq *$ if and only if the Alexandroff topology $\tau_{\leqq *}$ is equal to the quotient topology. In other words, if the decomposition space $X / \mathcal{D}=\Lambda$ with the quotient topology is an Alexandroff space, then the order $\leqq^{*}$ is the same as $\leqq_{\tau_{\pi_{\mathcal{D}}}}$, i.e., $\lambda \leqq \tau_{\pi_{\mathcal{D}}} \mu \Longleftrightarrow e_{\lambda} \subset \overline{e_{\mu}}$.

Such a continuous map from a topological space to a poset considered as a topological space with the Alexandroff topology has been studied in recent papers (e.g., $[4,9,23,35,41]$, etc.)

Definition 2.5. Let $P$ be a poset. A poset-stratified space $S$ over the poset $P$ is a pair $(S, S \stackrel{\pi}{\rightarrow} P)$ of a topological space $S$ and a continuous map $\pi: S \rightarrow P$ where $P$ is considered as the associated Alexandroff space.

Remark 2.6. The notion of poset-stratified space is due to Lurie [23]. For a posetstratified space $(S, S \stackrel{\pi}{\rightarrow} P), S$ is the underlying topological space and $\pi: S \rightarrow P$ is considered as a structure of poset-stratification. If the context is clear, then we just write a poset-stratified space $S$, just like writing a topological space $S$ without referring to which topology to be considered on it.

The category of poset-stratified spaces is denoted by Strat. The objects are pairs $(S, S \stackrel{\pi}{\rightarrow} P$ ) of a topological space $S$ and a continuous map $\pi: S \rightarrow P$ from the space $S$ 
to a poset $P$ with the Alexandroff topology associated to the poset $P$. Given two poset-stratified spaces $(S, S \stackrel{\pi}{\rightarrow} P)$ and $\left(S^{\prime}, S^{\prime} \stackrel{\pi^{\prime}}{\rightarrow} P\right)$, a morphism from $(S, S \stackrel{\pi}{\rightarrow} P)$ to $\left(S^{\prime}, S^{\prime} \stackrel{\pi^{\prime}}{\rightarrow} P^{\prime}\right)$ is a pair of a continuous map $f: S \rightarrow S^{\prime}$ and a monotone map $q: P \rightarrow P^{\prime}$ (i.e., for $a \leqq b$ in $P$ we have $q(a) \leqq q(b)$ in $P^{\prime}$, thus it is a continuous map for the associated Alexandroff spaces) such that the following diagram commutes:

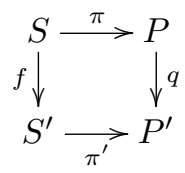

\section{A poset-stratified space structure of $[X, Y]$}

Lemma 3.1. On the homotopy set $[X, Y]$ we define the following orders, which are preorders:

1. $[f] \leqq_{R}[g] \Longleftrightarrow \exists[s] \in[X, X]$ such that $[f]=[g] \circ[s]$, i.e., the following diagram commutes up to homotopy:

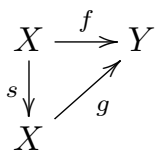

2. $[f] \leqq_{L}[g] \Longleftrightarrow \exists[t] \in[Y, Y]$ such that $[f]=[t] \circ[g]$, i.e., the following diagram commutes up to homotopy:

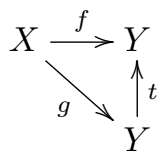

3. $[f] \leqq_{L R}[g] \Longleftrightarrow \exists[s] \in[X, X], \exists[t] \in[Y, Y]$ such that $[f]=[t] \circ[g] \circ[s]$, i.e., the following diagram commutes up to homotopy:

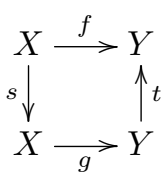

Lemma 3.2. On the homotopy set $[X, Y]$ we define the following relations:

1. right equivalence relation: $[f] \sim_{R}[g] \Longleftrightarrow[f] \leqq_{R}[g]$ and $[g] \leqq_{R}[f]$, i.e., $\exists\left[s_{1}\right],\left[s_{2}\right] \in[X, X]$ such that $[f]=[g] \circ\left[s_{1}\right],[g]=[f] \circ\left[s_{2}\right]$, i.e., the following diagram commutes up to homotopy:

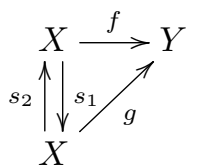

The relation $\sim_{R}$ is an equivalence relation and the set of equivalence classes 
shall be denoted by

$$
[X, Y]_{R}:=[X, Y] / \sim_{R}
$$

The equivalence class of $[f]$ is denoted by $[f]_{R}$.

2. left equivalence relation: $[f] \sim_{L}[g] \Longleftrightarrow[f] \leqq_{L}[g]$ and $[g] \leqq_{L}[f]$, i.e., $\exists\left[t_{1}\right]$, $\left[t_{2}\right] \in[Y, Y]$ such that $[f]=\left[t_{1}\right] \circ[g],[g]=\left[t_{2}\right] \circ[f]$, i.e., the following diagram commutes up to homotopy:

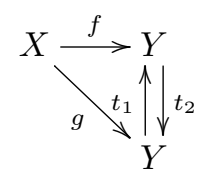

The relation $\sim_{L}$ is an equivalence relation and the set of equivalence classes shall be denoted by

$$
[X, Y]_{L}:=[X, Y] / \sim_{L} .
$$

The equivalence class of $[f]$ is denoted by $[f]_{L}$.

3. left-right equivalence relation $[f] \sim_{L R}[g] \Longleftrightarrow[f] \leqq_{L R}[g]$ and $[g] \leqq_{L R}[f]$, i.e.,

$$
\begin{aligned}
\exists\left[s_{1}\right],\left[s_{2}\right] \in[X, X], \exists\left[t_{1}\right],\left[t_{2}\right] \in[Y, Y] \text { such that }[f]= & {\left[t_{1}\right] \circ[g] \circ\left[s_{1}\right], } \\
& \text { and }[g]=\left[t_{2}\right] \circ[f] \circ\left[s_{2}\right]
\end{aligned}
$$

i.e., the following diagram commutes up to homotopy:

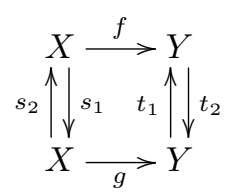

The relation $\sim_{L R}$ is an equivalence relation and the set of equivalence classes shall be denoted by

$$
[X, Y]_{L R}:=[X, Y] / \sim_{L R}
$$

The equivalence class of $[f]$ is denoted by $[f]_{L R}$.

Remark 3.3. As to the above relation $[f] \leqq_{L}[g]$, Stasheff (private communication) informed us of Borsuk's papers [6, 7] and Hilton's paper [18] (cf. [19, 20]). Borsuk introduced dependence of maps: $f: X \rightarrow Y$ is said to depend on $g: X \rightarrow Y$ if whenever $g$ is extended to $X^{\prime} \supset X$, so is $f$. He gave an alternative naming for this notion: $f$ is a multiple of $g$ or $g$ is a divisor of $f$. It turned out that this naming was correct, because Borsuk proved that $f$ depends on $g$ if and only if there exists a map $t: Y \rightarrow Y$ such that $f \sim t \circ g$, i.e., $[f] \leqq_{L}[g]$ in our notation. Furthermore Borsuk defined two maps $f$ and $g$ to be conjugate if they depend on each other, i.e., $[f] \sim_{L}[g]$ in our notation. Dually, $f: X \rightarrow Y$ is said to co-depend on $g: X \rightarrow Y$ if whenever $g$ lifts to the total space $E$ of a fibration over $Y$, so does $g$. Then the dual of the above Borsuk's result is that $f$ co-depends on $g$ if and only if there exists a map $s: X \rightarrow X$ such that $f \sim g \circ s$, i.e., $[f] \leqq_{R}[g]$ in our notion. Thus, using Borsuk's notion, $[X, Y]_{R}$ and $[X, Y]_{L}$ are the poset of the homotopy classes of co-conjugate maps and conjugate maps, resp. In 
this sense, $[X, Y]_{L R}$ is the poset of homotopy classes of conjugate-co-conjugate maps, abusing words. According to [19, 20], Thom [36] independently introduced the notion of dependence of cohomology classes, but it turned out that Thom's dependence is subsumed in Borsuk's dependence, and the above results about the co-dependence marked the birth of Eckmann-Hilton duality.

We can define orders on $[X, Y]_{R},[X, Y]_{L},[X, Y]_{L R}$. For the sake of completeness we write them down below.

Proposition 3.4. The following orders are well-defined and they are partial orders, i.e., reflexive, antisymmetric and transitive:

1. For $[f]_{R},[g]_{R} \in[X, Y]_{R},[f]_{R} \leqq_{R}^{\prime}[g]_{R} \Longleftrightarrow \exists[\phi] \in[X, X]$ such that $[f]=[g] \circ[\phi]$, i.e., the following diagram commutes up to homotopy (namely, $f \sim g \circ \phi$ ):

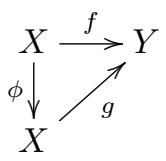

2. For $[f]_{L},[g]_{L} \in[X, Y]_{L},[f]_{L} \leqq_{L}^{\prime}[g]_{L} \Longleftrightarrow \exists[\psi] \in[Y, Y]$ such that $[f]=[\psi] \circ[g]$, i.e., the following diagram commutes up to homotopy (namely, $f \sim \psi \circ g$ ):

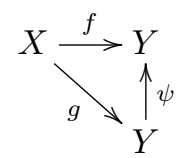

3. For $[f]_{L R},[g]_{L R} \in[X, Y]_{L R},[f]_{L R} \varliminf_{L R}^{\prime}[g]_{L R} \Longleftrightarrow \exists[\phi] \in[X, X], \exists[\psi] \in[Y, Y]$ such that $[f]=[\psi] \circ[g] \circ[\phi]$, i.e., the following diagram commutes up to homotopy (namely, $f \sim \psi \circ g \circ \phi$ ):

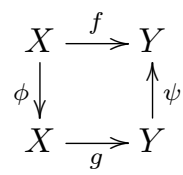

Proposition 3.5. The following canonical maps are monotone maps:

1. $\pi_{R}:\left([X, Y], \leqq_{R}\right) \rightarrow\left([X, Y]_{R}, \leqq_{R}^{\prime}\right), \pi_{R}([f]):=[f]_{R} ;$

2. $\pi_{L}:\left([X, Y], \leqq_{L}\right) \rightarrow\left([X, Y]_{L}, \leqq_{L}^{\prime}\right), \pi_{L}([f]):=[f]_{L}$;

3. $\pi_{L R}:\left([X, Y], \leqq_{L R}\right) \rightarrow\left([X, Y]_{L R}, \varliminf_{L R}^{\prime}\right), \pi_{L R}([f]):=[f]_{L R}$.

Hence each is a continuous map from a topological space (which is an Alexandroff space) to a poset with the Alexandroff topology. In other words the homotopy set $[X, Y]$ can have these three poset-stratified space structures.

Theorem 3.6. Let $h \mathcal{T}$ op be the homotopy category.

1. For any object $S \in O b j(h \mathcal{T} o p)$, we have an associated covariant functor $\mathfrak{s t}_{*}^{S}: h \mathcal{T}$ op $\rightarrow$ Strat such that

(a) for each object $Y \in \operatorname{Obj}(h \mathcal{T}$ op $)$,

$$
\mathfrak{s t}_{*}^{S}(X):=\left(\left([S, X], \tau_{\varliminf_{R}}\right),\left([S, X], \tau_{\varliminf_{R}}\right) \stackrel{\pi_{R}}{\longrightarrow}\left([S, X]_{R}, \varliminf_{R}^{\prime}\right)\right)
$$


(b) for a morphism $[f] \in[X, Y], \mathfrak{s t}_{*}^{S}([f])$ is the following commutative diagram:

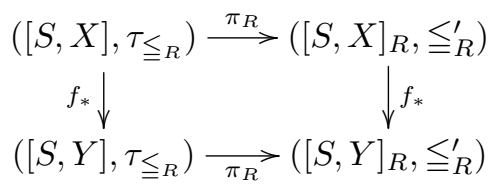

2. For any object $T \in \operatorname{Obj}(h \mathcal{T}$ op $)$, we have an associated contravariant functor $\mathfrak{s t}_{T}^{*}: h \mathcal{T}$ op $\rightarrow$ Strat such that

(a) for each object $X \in \operatorname{Obj}(h \mathcal{T}$ op $)$,

$$
\mathfrak{s t}_{T}^{*}(X):=\left(\left([X, T], \tau_{\varliminf_{L}}\right),\left([X, T], \tau_{\varliminf_{L}}\right) \stackrel{\pi_{L}}{\longrightarrow}\left([X, T]_{L}, \leqq_{L}^{\prime}\right)\right)
$$

(b) for a morphism $[f] \in[X, Y], \mathfrak{s t}_{T}^{*}([f])$ is the following commutative diagram:

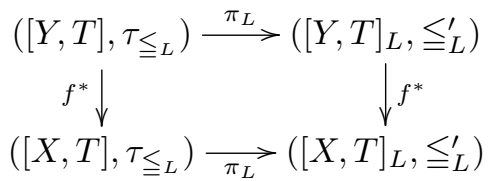

Example 3.7. Let $X=Y_{1}=K(\mathbb{Q}, 3) \times K(\mathbb{Q}, 2)$ and $Y_{2}=K(\mathbb{Q}, 3) \times K(\mathbb{Q}, 5)$. Recall the Sullivan minimal model $M(S)$ of a space $S[\mathbf{1 3}]$. Then homotopy sets are identified with DGA(differential graded algebra)-homotopy sets as

$$
\begin{aligned}
(1) & {\left[X, Y_{1}\right]=\left[M\left(Y_{1}\right), M(X)\right]=[(\Lambda(x, y), 0),(\Lambda(x, y), 0)], } \\
(2) \quad\left[X, Y_{2}\right] & =\left[M\left(Y_{2}\right), M(X)\right]=[(\Lambda(x, z), 0),(\Lambda(x, y), 0)],
\end{aligned}
$$

where $|x|=3,|y|=2$ and $|z|=5$. They are isomorphic to $\mathbb{Q} \times \mathbb{Q}=\{(a, b) \mid a, b \in \mathbb{Q}\}$ by the DGA-maps $f(x)=a x$ and $f(y)=b y$ for $(1)$ and $f(x)=a x$ and $f(z)=b x y$ for (2), respectively. Then their right equivalence classes are (1) $\left[X, Y_{1}\right]_{R}=\{\alpha, \beta, \gamma, \delta\}$ and $(2)\left[X, Y_{2}\right]_{R}=\left\{\alpha^{\prime}, \beta^{\prime}, \gamma^{\prime}, \delta^{\prime}\right\}$ where $\alpha=\alpha^{\prime}=[(0,0)]_{R}, \beta=\beta^{\prime}=[(1,0)]_{R}, \gamma=\gamma^{\prime}$ $=[(0,1)]_{R}$ and $\delta=\delta^{\prime}=[(1,1)]_{R}$. However, their poset structures are given as the following Hasse diagrams:

$(1)$

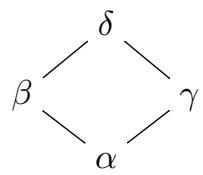

$(2)$

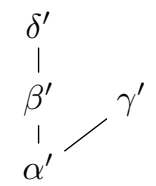

respectively. In particular, there does not exist $\gamma^{\prime} \leqq_{R}^{\prime} \delta^{\prime}$ in $(2)$ since $\psi(M(f)(z))=$ $\psi(x y)=0$ if $\psi(M(f)(x))=\psi(x)=0$ for $\psi: M(X) \rightarrow M(X)$. For both cases, the stratifications of $\mathbb{Q} \times \mathbb{Q}$ are given as

$$
\mathbb{Q} \times \mathbb{Q}=e_{\alpha} \cup e_{\beta} \cup e_{\gamma} \cup e_{\delta}=e_{\alpha^{\prime}} \cup e_{\beta^{\prime}} \cup e_{\gamma^{\prime}} \cup e_{\delta^{\prime}},
$$

where $e_{\alpha}=e_{\alpha^{\prime}}=\{(0,0)\}, e_{\beta}=e_{\beta^{\prime}}=\{(a, 0) \mid a \neq 0\}, e_{\gamma}=e_{\gamma^{\prime}}=\{(0, b) \mid b \neq 0\}$ and $e_{\delta}=e_{\delta^{\prime}}=\{(a, b) \mid a b \neq 0\}$. However, the topologies are different. Indeed, $\overline{e_{\delta}}=\mathbb{Q} \times \mathbb{Q}$ in (1) but $\overline{e_{\delta^{\prime}}}$ does not contain $e_{\gamma^{\prime}}$ in $(2)$.

If a map $f: Y_{1} \rightarrow Y_{2}$ is given by $M(f):(\Lambda(x, z), 0) \rightarrow(\Lambda(x, y), 0)$ with $M(f)(x)=x$ and $M(f)(z)=x y$, the induced map of homotopy sets $f_{*}:\left[X, Y_{1}\right]=\mathbb{Q} \times \mathbb{Q} \rightarrow\left[X, Y_{2}\right]$ $=\mathbb{Q} \times \mathbb{Q}$ is given by $f_{*}(a, b)=(a, a b)$. Then the poset map $f_{*}:\left[X, Y_{1}\right]_{R} \rightarrow\left[X, Y_{2}\right]_{R}$ is given by $f_{*}(\alpha)=f_{*}(\gamma)=\alpha^{\prime}, f_{*}(\beta)=\beta^{\prime}$ and $f_{*}(\delta)=\delta^{\prime}$. 


\section{Some applications}

Definition 4.1. For a group $G$ let $\mathcal{S} u b(G)$ be the set of all the subgroups of the group $G$. For subgroups $A, B \in \mathcal{S} u b(G)$ we define the order $A \leqq B$ by $A \subseteq B$, which is a partial order.

Lemma 4.2. Let $H_{*}(-)$ be the homology theory with a coefficient ring $\mathscr{R}$. Then the following maps are well-defined and monotone (order-preserving) maps:

1. $\operatorname{Im}_{H_{*}}:\left([X, Y], \leqq_{R}\right) \rightarrow\left(\mathcal{S u b}\left(H_{*}(Y)\right), \leqq\right)$,

$\operatorname{Im}_{H_{*}}([f]):=\operatorname{Im}\left(f_{*}: H_{*}(X) \rightarrow H_{*}(Y)\right)$.

2. $\operatorname{Im}_{H_{*}}^{\prime}:\left([X, Y]_{R}, \varliminf_{R}^{\prime}\right) \rightarrow\left(\mathcal{S} u b\left(H_{*}(Y)\right), \leqq\right), \operatorname{Im}_{H_{*}}^{\prime}\left([f]_{R}\right):=\operatorname{Im}_{H_{*}}([f])$.

We have the following commutative diagram:

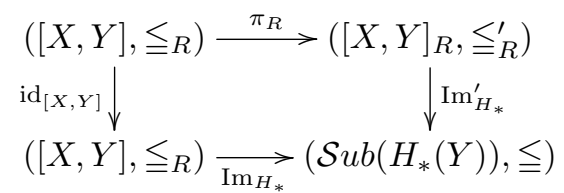

Proof. Let $[f] \leqq_{R}[g]$. Thus $\exists t: X \rightarrow X$ such that $f \sim g \circ t$. Hence $f_{*}=g_{*} \circ t_{*}$, i.e., the following diagram commutes:

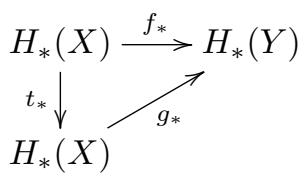

which implies that $\operatorname{Im}\left(f_{*}: H_{*}(X) \rightarrow H_{*}(Y)\right) \subset \operatorname{Im}\left(g_{*}: H_{*}(X) \rightarrow H_{*}(Y)\right)$. Thus $\operatorname{Im}_{H_{*}}([f]) \subset \operatorname{Im}_{H_{*}}([g])$. Hence $\operatorname{Im}_{H_{*}}:\left([X, Y], \leqq_{R}\right) \rightarrow\left(\mathcal{S} u b\left(H_{*}(Y)\right), \leqq\right)$ is a monotone map. For $\operatorname{Im}_{H_{*}}^{\prime}$ we just observe that if $[f] \sim_{R}[g]$, i.e., $\exists t_{1}: X \rightarrow X, t_{2}: X \rightarrow X$ such that $f \sim g \circ t_{1}$ and $g \sim g \circ t_{2}$, then it follows from the above that $\operatorname{Im}\left(f_{*}: H_{*}(X) \rightarrow\right.$ $\left.H_{*}(Y)\right)=\operatorname{Im}\left(g_{*}: H_{*}(X) \rightarrow H_{*}(Y)\right)$, i.e., $\operatorname{Im}_{H_{*}}([f])=\operatorname{Im}_{H_{*}}([g])$. Thus $\operatorname{Im}_{H_{*}}\left([f]_{R}\right):=$ $\operatorname{Im}_{H_{*}}([f])$ is well-defined.

Similarly we get the following:

Lemma 4.3. Let $H^{*}(-)$ be the cohomology theory with a coefficient ring $\mathscr{R}$. Then the following maps are well-defined and monotone (order-preserving) maps:

1. $\operatorname{Im}_{H^{*}}:\left([X, Y], \leqq_{L}\right) \rightarrow\left(\mathcal{S} u b\left(H^{*}(X)\right), \leqq\right)$, $\operatorname{Im}_{H^{*}}([f]):=\operatorname{Im}\left(f^{*}: H^{*}(Y) \rightarrow H_{*}(X)\right)$.

2. $\operatorname{Im}_{H^{*}}^{\prime}:\left([X, Y]_{L}, \varliminf_{L}^{\prime}\right) \rightarrow\left(\mathcal{S} u b\left(H^{*}(X)\right), \leqq\right), \operatorname{Im}_{H^{*}}^{\prime}\left([f]_{L}\right):=\operatorname{Im}_{H^{*}}([f])$.

We have the following commutative diagram:

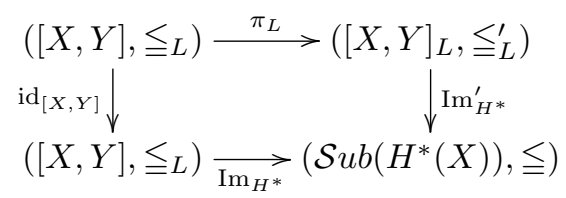

Corollary 4.4. Let $H_{*}(-)$ and $H^{*}(-)$ be as above.

1. For $\forall S \in O b j(h \mathcal{T}$ op $)$, we have a covariant functor $\mathfrak{s t}_{H_{*}}^{S}: h \mathcal{T}$ op $\rightarrow$ Strat such that 
(a) for each object $X \in \operatorname{Obj}(h \mathcal{T}$ op $)$,

$$
\mathfrak{s t}_{H_{*}}^{S}(X):=\left(\left([S, X], \tau_{\varliminf_{R}}\right),\left([S, X], \tau_{\varliminf_{R}}\right) \stackrel{\operatorname{Im}_{H_{*}}}{\longrightarrow}\left(\mathcal{S} u b\left(H_{*}(X)\right), \leqq\right)\right)
$$

(b) for a morphism $[f] \in[X, Y], \mathfrak{s t}_{H_{*}}^{S}([f])$ is the following commutative diagram:

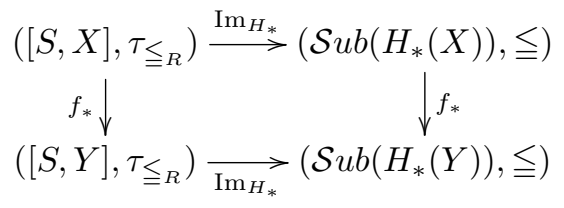

2. $\operatorname{Im}_{H_{*}}^{\prime}$ gives rise to a natural transformation $\operatorname{Im}_{H_{*}}^{\prime}: \mathfrak{s t}_{*}^{S}(-) \rightarrow \mathfrak{s t}_{H_{*}}^{S}(-)$, namely for a morphism $[f] \in[X, Y]$ we have the following commutative diagram:

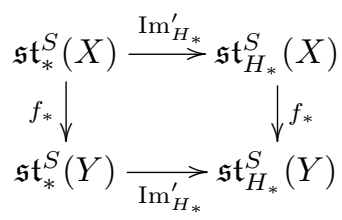

Namely we have the following commutative cube:

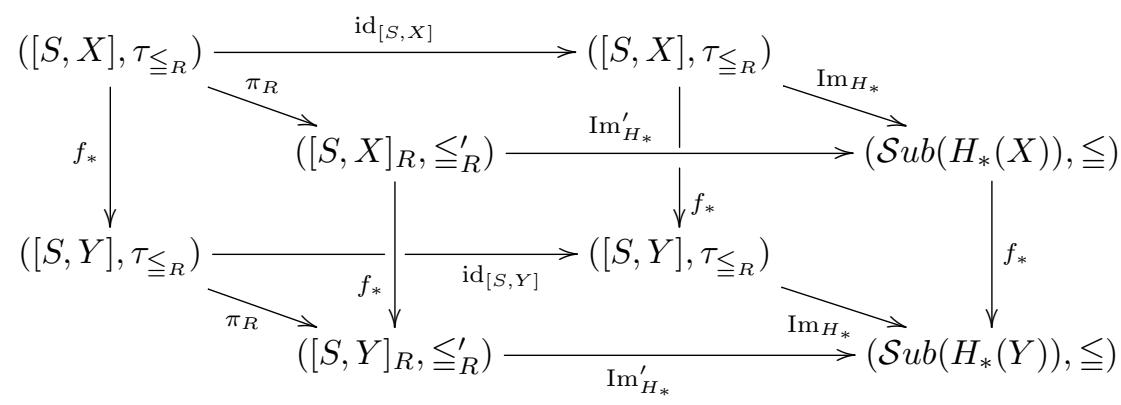

3. For any object $T \in O b j(h \mathcal{T} o p)$, we have an associated contravariant functor $\mathfrak{s t}_{T}^{H^{*}}: h \mathcal{T}$ op $\rightarrow$ Strat such that

(a) for each object $X \in \operatorname{Obj}(h \mathcal{T} o p)$,

$$
\mathfrak{s t}_{T}^{H^{*}}(X):=\left(\left([X, T], \tau_{\varliminf_{L}}\right),\left([X, T], \tau_{\leqq L}\right) \stackrel{\operatorname{Im}_{H^{*}}}{\longrightarrow}\left(\mathcal{S} u b\left(H^{*}(X)\right), \leqq\right)\right)
$$

(b) for a morphism $[f] \in[X, Y], \mathfrak{s t}_{T}^{H^{*}}([f])$ is the following commutative diagram:

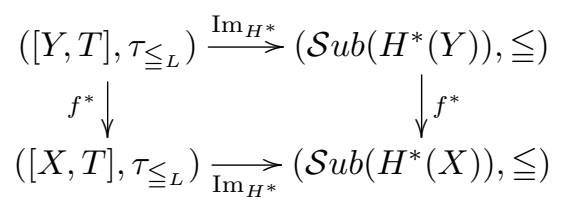

4. $\operatorname{Im}_{H^{*}}^{\prime}$ gives rise to a natural transformation $\operatorname{Im}_{H^{*}}^{\prime}: \mathfrak{s t}_{T}^{*}(-) \rightarrow \mathfrak{s t}_{T}^{H^{*}}(-)$, namely for a morphism $[f] \in[X, Y]$ we have the following commutative diagram: 


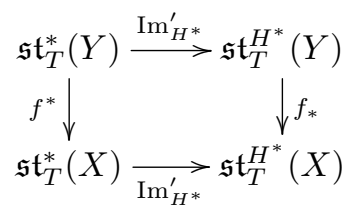

Namely we have the following commutative cube:

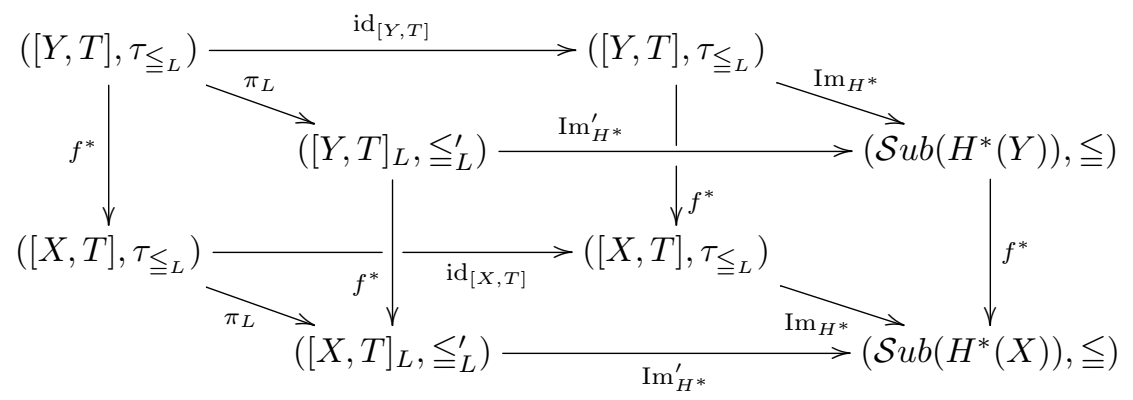

The case of $\operatorname{Im}_{H^{*}}:\left([X, T], \leqq_{L}\right) \rightarrow\left(\mathcal{S} u b\left(H^{*}(X), \leqq\right)\right.$ is related to Thom's dependence of cohomology classes [36] mentioned in the introduction. To explain this, we recall the definition of dependence of cohomology classes (e.g., see [18]).

Definition 4.5 (R. Thom). The cohomology class $\beta \in H^{q}(X ; B)$ depends on the cohomology class $\alpha \in H^{p}(X ; A)$, where $A, B$ are coefficient rings, if, for all (perhaps infinite) polyhedra $Y$ and all maps $f: X \rightarrow Y$ such that $\alpha \in f^{*}\left(H^{p}(Y ; A)\right)$, we have $\beta \in f^{*}\left(H^{q}(Y ; B)\right)$.

Thom [36] proves the following proposition (see [18]). For this we recall that the cohomology theory is representable by the Eilenberg-Maclane space, i.e., $H^{j}(X, \Lambda) \cong$ $[X, K(\Lambda, j)]$ where $K(R, j)$ is the Eilenberg-Maclane space whose homotopy type is completely characterized by the homotopy groups $\pi_{j}(K(\Lambda, j))=\Lambda$ and $\pi_{i}(K(\Lambda, j))=$ $0, i \neq j$. Then by the Hurewicz Theorem we have $H_{j}(K(\Lambda, j) ; \mathbb{Z}) \cong \pi_{j}(K(\Lambda, j))=\Lambda$ and $H_{d}(K(\Lambda, j))=0$ for $d<j$. Hence by the universal coefficient theorem we have the isomorphism

$$
\begin{aligned}
\Phi: H^{j}(K(\Lambda, j) ; \Lambda) & \cong \operatorname{Hom}\left(H_{j}(K(\Lambda, j) ; \mathbb{Z}), \Lambda\right) \\
& \cong \operatorname{Hom}\left(\pi_{j}(K(\Lambda, j)), \Lambda\right) \cong \operatorname{Hom}(\Lambda, \Lambda) .
\end{aligned}
$$

Let $u:=\Phi^{-1}\left(\operatorname{id}_{\Lambda}\right)$ for the identity $\operatorname{map} \operatorname{id}_{\Lambda}: \Lambda \rightarrow \Lambda$. Then the isomorphism

$$
\Theta:[X, K(\Lambda, j)] \cong H^{j}(X, \Lambda)
$$

is obtained by $\Theta([f]):=f^{*} u$ where $f^{*}: H^{j}(K(\Lambda, j) ; \Lambda) \rightarrow H^{j}(X, \Lambda)$.

Proposition 4.6 (Thom [36]). Let $\alpha \in H^{p}(X ; A) \cong[X, K(A, p)]$ and let $f_{\alpha}: X \rightarrow$ $K(A, p)$ be a map such that the homotopy class $\left[f_{\alpha}\right]$ corresponds to $\alpha$. Then $\beta \in$ $H^{q}(X, B)$ depends on $\alpha$ if and only if $\beta \in f_{\alpha}^{*}\left(H^{q}(K(A, p) ; B)\right)$.

Using this proposition we can get the following result. By the monotone (orderpreserving) map

$$
\operatorname{Im}_{H^{*}}:\left([X, K(A, p)], \varliminf_{L}\right) \rightarrow\left(\mathcal{S} u b\left(H^{*}(X ; B)\right), \leqq\right)
$$

the image $\operatorname{Im}_{H^{*}}\left(\left[f_{\alpha}\right]\right)=f_{\alpha}^{*}\left(H^{q}(K(A, p) ; B)\right)$ is nothing but the subgroup of all the 
cohomology classes $\beta \in H^{q}(X ; B)$ depending on the cohomology class $\alpha$.

We also see that let $\alpha, \alpha^{\prime} \in H^{p}(X, A)$ and let $f_{\alpha}, f_{\alpha^{\prime}}: X \rightarrow K(A, p)$ be the corresponding maps. Then, if $f_{\alpha}$ depends on $f_{\alpha^{\prime}}$, i.e., $\left[f_{\alpha}\right] \leqq_{L}\left[f_{\alpha^{\prime}}\right]$ by our terminology (in other words, we can define the order of the cohomology classes $\alpha \leqq_{L} \alpha^{\prime}$ by this), then we have $(\alpha \in) \operatorname{Im}_{H^{*}}\left(\left[f_{\alpha}\right]\right) \subset \operatorname{Im}_{H^{*}}\left(\left[f_{\alpha^{\prime}}\right]\right)$, i.e., $\operatorname{Im}_{H^{*}}\left(\left[f_{\alpha}\right]\right) \leqq \operatorname{Im}_{H^{*}}\left(\left[f_{\alpha^{\prime}}\right]\right)$. Thus, that $\alpha$ depends on $\alpha^{\prime}$ is equivalent to that $\operatorname{Im}_{H^{*}}\left(\left[f_{\alpha}\right]\right) \leqq \operatorname{Im}_{H^{*}}\left(\left[f_{\alpha^{\prime}}\right]\right)$.

Here is another application to vector bundles and characteristic classes (e.g., see $[31,17])$. Let $\operatorname{Vect}_{n}(X)$ be the set of isomorphism classes of complex vector bundles of rank $n$. Then it is well-known that

$$
\operatorname{Vect}_{n}(X) \cong\left[X, G_{n}\left(\mathbb{C}^{\infty}\right)\right]
$$

where $G_{n}\left(\mathbb{C}^{\infty}\right)$ is the infinite Grassmann manifold of complex planes of dimension $n$, i.e., the classifying space of complex vector bundles of rank $n$. This isomorphism is by the correspondence $[E] \longleftrightarrow\left[f_{E}\right]$, where $f_{E}: X \rightarrow G_{n}\left(\mathbb{C}^{\infty}\right)$ is a classifying map of $E$, i.e., $E=f_{E}^{*} \gamma^{n}$, where $\gamma^{n}$ is the universal complex vector bundle of rank $n$ over $G_{n}\left(\mathbb{C}^{\infty}\right)$.

By the isomorphism $\operatorname{Vect}_{n}(X) \cong\left[X, G_{n}\left(\mathbb{C}^{\infty}\right)\right]$ we can consider the preorder of $[E]$ and $[F]$ :

$$
[E] \leqq_{L}[F] \Longleftrightarrow\left[f_{E}\right] \leqq_{L}\left[f_{F}\right],
$$

where $f_{E}, f_{F}: X \rightarrow G_{n}\left(\mathbb{C}^{\infty}\right)$ are respectively the classifying maps of $E$ and $F$.

Then we have the following well-defined monotone (order-preserving) map:

$$
\operatorname{Im}_{H^{*}}:\left(\operatorname{Vect}_{n}(X), \leqq_{L}\right) \rightarrow\left(\mathcal{S} u b\left(H^{*}(X ; \mathbb{Z})\right), \leqq\right)
$$

defined by $\operatorname{Im}_{H^{*}}([E]):=\operatorname{Im}\left(f_{E}^{*}: H^{*}\left(G_{n}\left(\mathbb{C}^{\infty}\right) ; \mathbb{Z}\right) \rightarrow H^{*}(X ; \mathbb{Z})\right)$. By the definition of characteristic classes, for each element $\alpha \in H^{*}\left(G_{n}\left(\mathbb{C}^{\infty}\right)\right)$, the pullback $f_{E}^{*}(\alpha)$ is called the characteristic class of $E$ defined by the class $\alpha$, and denoted by $\alpha(E):=f_{E}^{*}(\alpha)$. It is well-known (e.g., see [31]) that $H^{*}\left(G_{n}\left(\mathbb{C}^{\infty}\right)\right)=\mathbb{Z}\left[c_{1}, c_{2}, \ldots, c_{n}\right]$ is generated by 1 and the Chern classes $c_{1}, c_{2}, \ldots, c_{n}$ of the universal bundle $\gamma^{n}$. Here $1, c_{1}, c_{2}, \ldots, c_{n}$ are linearly independent. $\operatorname{Im}\left(f_{E}^{*}: H^{*}\left(G_{n}\left(\mathbb{C}^{\infty}\right) ; \mathbb{Z}\right) \rightarrow H^{*}(X ; \mathbb{Z})\right)$ is nothing but the subring consisting of all the characteristic classes of $E$, which could be also denoted by

$$
\mathbb{Z}\left[c_{1}(E), c_{2}(E), \ldots, c_{n}(E)\right] .
$$

Here we should note that $1, c_{1}(E), c_{2}(E), \ldots, c_{n}(E)$ are not linearly independent in general. Let us denote this subring by $\operatorname{Char}(E)$. Therefore we have $[E] \leqq_{L}[F] \Longrightarrow$ $\operatorname{Char}(E) \subseteq \operatorname{Char}(F)$. We also get that $[E] \sim_{L}[F] \Longrightarrow \operatorname{Char}(E)=\operatorname{Char}(F)$.

Remark 4.7. In the case of real vector bundles, the complex infinite Grassmann $G_{n}\left(\mathbb{C}^{\infty}\right)$, the Chern class $c_{i}$ and the coefficient ring $\mathbb{Z}$ are respectively replaced by the real infinite Grassmann $G_{n}\left(\mathbb{R}^{\infty}\right)$, the Stiefell-Whitney class $w_{i}$ and the coefficient $\operatorname{ring} \mathbb{Z}_{2}$.

Remark 4.8. Instead of homology $H_{*}(-)$ and cohomology $H^{*}(-)$, we can consider homotopy version of these, i.e., homotopy groups $\pi_{*}(-)$ and cohomotopy "groups" $\pi^{*}(-)$. In this case we consider the based homotopy set $[X, Y]_{*}$. We note that the cohomotopy set $\pi^{p}(X):=\left[X, S^{p}\right]$ (e.g., see [22]). Note that in the case when $p=1$, $\pi^{1}(X)=\left[X, S^{1}\right]=[X, K(\mathbb{Z}, 1)]=H^{1}(X ; \mathbb{Z})$ is an abelian group. 
Remark 4.9. For any locally small category $\mathcal{C}$, in a similar manner as above we can consider a poset-stratified space structure on the hom set $h_{\mathcal{C}}(X, Y)$ for any objects $X, Y \in \operatorname{Obj}(\mathcal{C})$, and using reasonable covariant functor $\mathcal{H}_{*}$ and contravariant functor $\mathcal{H}^{*}$ on the locally small category $\mathcal{C}$ we can do similar things as above. For example, derived categories, triangulated categories, and derived functors, etc.

When it comes to the homotopy groups $\pi_{*}$, we have another application. We let $\operatorname{Map}(X, Y ; f)$ be the path component of $\operatorname{Map}(X, Y)$ containing $f$. Let $*$ be the base point of $X$ and we consider the evaluation map

$$
e v: \operatorname{Map}(X, Y ; f) \rightarrow Y, \quad e v(g):=g(*) .
$$

Definition 4.10 ([38]). For a continuous based map $f: X \rightarrow Y$, the $n$-th evaluation subgroup $G_{n}(Y, X ; f)$ of the $n$-th homotopy group $\pi_{n}(Y)$ is defined as follows:

$$
G_{n}(Y, X ; f):=\operatorname{Im}\left(e v_{*}: \pi_{n}(M a p(X, Y ; f)) \rightarrow \pi_{n}(Y)\right) .
$$

This is a generalized version of the following Gottlieb group $G_{n}(X)[\mathbf{1 5}, \mathbf{1 6}]$ :

$$
G_{n}(X):=\operatorname{Im}\left(e v_{*}: \pi_{n}\left(a u t_{1} X\right) \rightarrow \pi_{n}(X)\right),
$$

where $\operatorname{aut}_{1} X=\operatorname{Map}\left(X, X ; \operatorname{id}_{X}\right)$ and $i d_{X}$ is the identity map.

The $n$-th evaluation subgroup $G_{n}(Y, X ; f)$ can be described as follows:

Lemma 4.11 ([38]). The $n$-th evaluation subgroup of a continuous based map $f: X \rightarrow Y$ is

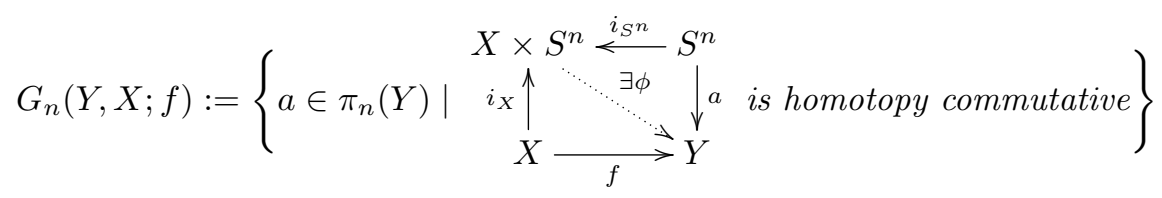

from the adjointness.

As to the case of generalized Gottlieb groups, we need to reverse the order.

Proposition 4.12. The following map (called "the $n$-th generalized Gottlieb evaluation subgroup map")

$$
\mathfrak{g}_{n}:[X, Y] \rightarrow \mathcal{S}\left(\pi_{n}(Y)\right) \quad G_{n}([f]):=G_{n}(Y, X ; f)
$$

is well-defined, i.e., $f \sim f^{\prime}$ implies that $G_{n}(Y, X ; f)=G_{n}\left(Y, X ; f^{\prime}\right)$.

Proposition 4.13. The following map (called "the finer $n$-th generalized Gottlieb evaluation subgroup map")

$$
\mathfrak{g}_{n}^{R}:[X, Y]_{R} \rightarrow \mathcal{S}\left(\pi_{n}(Y)\right) \quad \mathfrak{g}_{n}^{R}\left([f]_{R}\right):=G_{n}(Y, X ;[f])=G_{n}(Y, X ; f)
$$

is well-defined, i.e., $[f] \sim_{R}[g]$ implies that $G_{n}(Y, X ; f)=G_{n}(Y, X ; g)$. Namely the following diagram commutes:

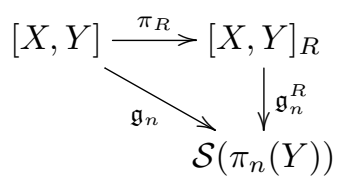


Proof. For two maps $f, g: X \rightarrow Y$, suppose that $f \sim g \circ h$ for some map $s: X \rightarrow X$. Then $G_{n}(Y, X ; g) \subset G_{n}(Y, X ; f)$. Indeed, there is the homotopy commutative diagram for $a \in G_{n}(Y, X ; g)$ :

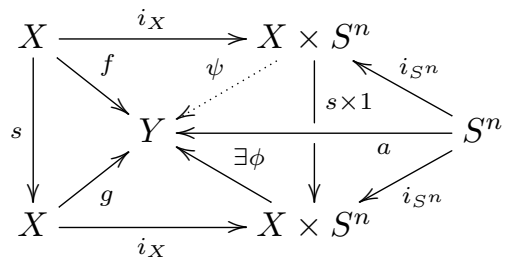

by $\psi:=\phi \circ(s \times 1)$. Then $\psi \circ i_{X} \simeq f$ and $\psi \circ i_{S^{n}} \simeq a$. Hence $a \in G_{n}(Y, X ; f)$. Moreover, suppose that $g \sim f \circ s^{\prime}$. Then similarly we obtain $G_{n}(Y, X ; f) \subset G_{n}(Y, X ; g)$.

As a corollary of the above proof, we have the following:

\section{Theorem 4.14.}

1. If $[f] \leqq_{R}[g]$, then we have $G_{n}(Y, X ; g) \subset G_{n}(Y, X ; f)$, i.e., $\mathfrak{g}_{n}([g]) \leqq \mathfrak{g}_{n}([f])$. Hence $\mathfrak{g}_{n}:\left([X, Y], \leqq_{R}^{o p}\right) \rightarrow \mathcal{S} u b\left(\pi_{n}(Y), \leqq\right)$ is a monotone map.

2. If $[f]_{R} \leqq[g]_{R}$, then we have $G_{n}(Y, X ; g) \subset G_{n}(Y, X ; f)$, i.e., $\mathfrak{g}_{n}^{R}\left([g]_{R}\right) \leqq \mathfrak{g}_{n}^{R}\left([f]_{R}\right)$. Hence $\mathfrak{g}_{n}^{R}:\left([X, Y]_{R}, \leqq_{R}^{\prime o p}\right) \rightarrow \mathcal{S} u b\left(\pi_{n}(Y), \leqq\right)$ is a monotone map.

We also have the following commutative diagram:

$$
\begin{aligned}
& \left([X, Y], \leqq_{R}^{o p}\right) \stackrel{\pi_{R}}{\longrightarrow}\left([X, Y]_{R}, \leqq_{R}^{\prime o p}\right) \\
& \operatorname{id}_{[X, Y]} \downarrow \\
& \left([X, Y], \varliminf_{R}^{o p}\right) \underset{\mathfrak{g}_{n}}{\longrightarrow}\left(\mathcal{S} u b\left(\pi_{n}(Y)\right), \leqq\right)
\end{aligned}
$$

\section{Corollary 4.15.}

1. For $\forall S \in O b j(h \mathcal{T}$ op $)$, we have a covariant functor $\mathfrak{s t}_{\text {Gott }}^{S}: h \mathcal{T}$ op $\rightarrow$ Strat such that

(a) for each object $X \in \operatorname{Obj}(h \mathcal{T}$ op $)$,

$$
\mathfrak{s t}_{\text {Gott }}^{S}(X):=\left(\left([S, X], \tau_{\varliminf_{R}^{o p}}\right),\left([S, X], \tau_{\varliminf_{R}^{o p}}\right) \stackrel{\mathfrak{g}_{n}}{\longrightarrow}\left(\mathcal{S} u b\left(\pi_{n}(X)\right), \leqq\right)\right)
$$

(b) for a morphism $[f] \in[X, Y], \mathfrak{s t}_{\text {Gott }}^{S}([f])$ is the following commutative diagram:

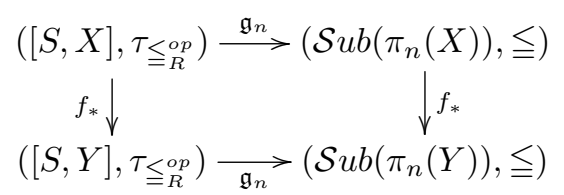

2. $\mathfrak{g}_{n}^{R}$ gives rise to a natural transformation $\mathfrak{g}_{n}^{R}: \mathfrak{s t}_{*}^{S}(-) \rightarrow \mathfrak{s t}_{\text {Gott }}^{S}(-)$, namely for a morphism $[f] \in[X, Y]$ we have the following commutative diagram:

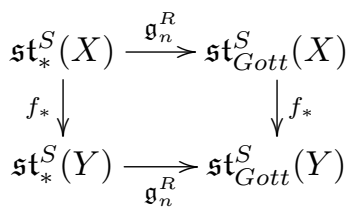


Namely we have the following commutative cube:

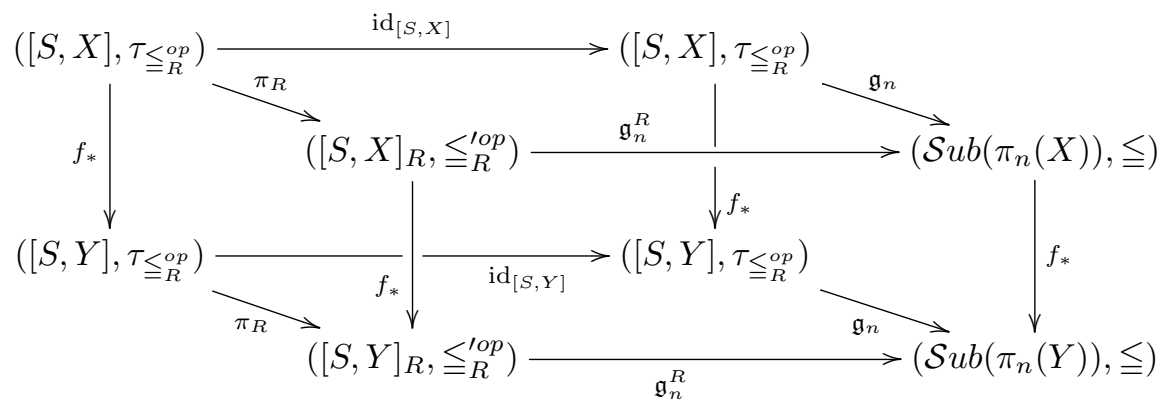

Remark 4.16. When it comes to the case $[X, Y]_{L}$ we do not have similar results as above.

Let $G_{*}(Y, X ; f):=\bigoplus_{n} G_{n}(Y, X ; f) \subset \pi_{*}(Y):=\bigoplus \pi_{n}(Y)$. We let

$$
\mathcal{G}(X, Y):=\left\{G_{*}(Y, X ; f) \mid f \in \operatorname{Map}(X, Y)\right\}
$$

be the poset with the partial order by the inclusions $G_{*}(Y, X ; g) \subset G_{*}(Y, X ; f)$ for some maps $f$ and $g$ from $X$ to $Y$. Then $\pi_{*}(Y)=G_{*}(Y, X ; *)$ is the maximal element of $\mathcal{G}(X, Y)$. In particular, when $X=Y$, the Gottlieb group $G_{*}(X):=G_{*}\left(X, X ; i d_{X}\right)$ is the minimal element of $\mathcal{G}(X, X)$. Thus

Corollary 4.17. The map $G:\left([X, Y]_{R}, \leqq_{R}^{\prime o p}\right) \rightarrow(\mathcal{G}(X, Y), \leqq)$ given by $G\left([f]_{R}\right)=$ $G(f):=G_{*}(Y, X ; f)$ is a poset map.

Example 4.18. Let $X=S^{n}$ and $Y=\left(S^{n} \times S^{n}\right)_{0}$ for an even integer $n$. Here $\left(S^{n} \times\right.$ $\left.S^{n}\right)_{0}$ is the rationalization of $S^{n} \times S^{n}[21]$. Then $[X, Y]_{R}=\mathbb{Q} \oplus \mathbb{Q} / \sim_{R}=P^{1}(\mathbb{Q}) \cup$ $(0,0)$ as a set with $(a, b) \sim_{R}\left(a^{\prime}, b^{\prime}\right)$ when $a^{\prime}=k a$ and $b^{\prime}=k b$ for some $k \in \mathbb{Q}-0$. It is ordered only by $[a, b]<(0,0)$ for any $[a, b] \in P^{1}(\mathbb{Q})$. On the other hand, $\mathcal{G}(X, Y)$ is the set of four points whose order is given as the Hasse diagram:

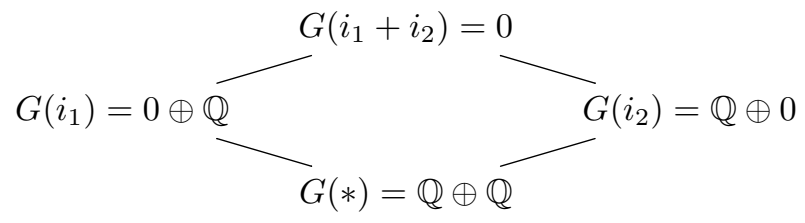

for the $k$-factor inclusion $i_{k}: S^{n} \rightarrow\left(S^{n} \times S^{n}\right)_{0}$ and the constant map $*$. Then the poset map $G:[X, Y]_{R} \rightarrow \mathcal{G}(X, Y)$ is given by $G((0,0))=\mathbb{Q} \oplus \mathbb{Q}, G([1,0])=0 \oplus \mathbb{Q}$, $G([0,1])=\mathbb{Q} \oplus 0$ and $G([a, b])=0$ when $a b \neq 0$.

Definition 4.19 ([42, Definition 2.1]). The $n$-th generalized dual Gottlieb set of a map $f: X \rightarrow Y$ is

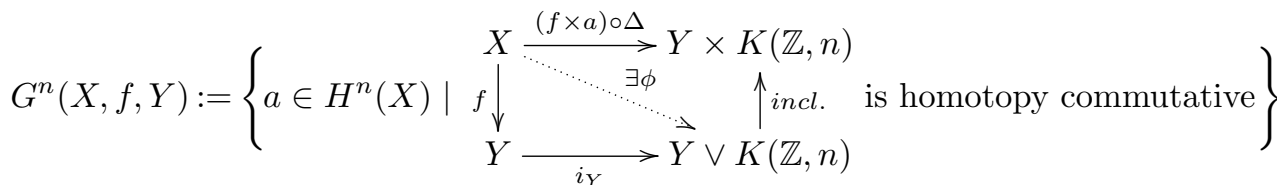

for the diagonal map $\Delta: X \rightarrow X \times X$. 
Proposition 4.20. The following map (called "the finer $n$-th generalized dual Gottlieb map")

$$
\mathfrak{g}_{L}^{n}:[X, Y]_{L} \rightarrow \mathcal{S}\left(H^{n}(X)\right) \quad \mathfrak{g}_{L}^{n}\left([f]_{L}\right):=G^{n}(X, f, Y)
$$

is well-defined, i.e., $[f] \sim_{L}[g]$ implies that $G^{n}(X, f, Y)=G^{n}(X, g, Y)$. Namely the following diagram commutes:

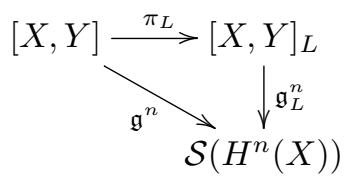

Proof. For two maps $f, g: X \rightarrow Y$, suppose that $g \sim s \circ f$ for some map $s: Y \rightarrow Y$. Then $G^{n}(X, f, Y) \subset G^{n}(X, g, Y)$. Indeed, there is the homotopy commutative diagram for $a \in G^{n}(X, f, Y)$ :

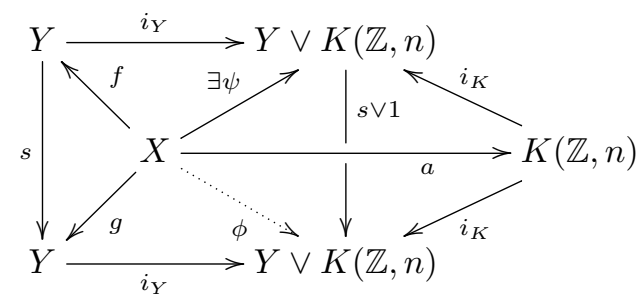

by $\phi:=(s \vee 1) \circ \psi$. Then $i_{X} \circ g \simeq \phi$ and $i_{S^{n}} \circ a \simeq \phi$. Hence $a \in G^{n}(X, g, Y)$. Furthermore, suppose that $f \sim s^{\prime} \circ g$. Then similarly we obtain $G^{n}(X, g, Y) \subset G^{n}(X, f, Y)$.

Remark 4.21. For generalized dual Gottlieb sets, we obtain similar properties as evaluation subgroups.

Example 4.22. Let cat $(f)$ be the Lusternik-Schnirelmann category of a map $f: X \rightarrow$ $Y\left[13\right.$, p. 352]. Then cat: $[X, Y] \rightarrow\left(\mathbb{Z}_{\geqq 0}, \leqq\right)$ is a monotone map. In the case of cat, we have the three finer poset-stratified space structure on the reversed ordered posets $[X, Y]_{R},[X, Y]_{L}$ and $[X, Y]_{L R}$ as follows:

1. If $[g] \leqq_{R}[f]$, i.e., $g \sim f \circ s$ with $s: X \rightarrow X$, then we have [13, Lemma 27.1(ii)]

$$
\operatorname{cat}(g)=\operatorname{cat}(f \circ s) \leqq \min \{\operatorname{cat}(f), \operatorname{cat}(s)\} \leqq \operatorname{cat}(f) \text {. }
$$

Hence we have $\operatorname{cat}(g) \leqq \operatorname{cat}(f)$. So there is a poset map $\operatorname{cat}_{R}:[X, Y]_{R} \rightarrow$ $\left(\mathbb{Z}_{\geqq 0}, \leqq\right)$. Here $\operatorname{cat}_{R}\left([f]_{R}\right):=\operatorname{cat}(f)$.

2. If $[g] \leqq_{L}[f]$, i.e., $g \sim t \circ f$ with $t: Y \rightarrow Y$, then we have

$$
\operatorname{cat}(g)=\operatorname{cat}(t \circ f) \leqslant \min \{\operatorname{cat}(t), \operatorname{cat}(f)\} \leqq \operatorname{cat}(f) .
$$

Hence we have cat $(g) \leqq \operatorname{cat}(f)$. Thus $\operatorname{cat}_{L}:[X, Y]_{L} \rightarrow\left(\mathbb{Z}_{\geqq 0}, \leqq\right)$ is a poset map. Here $\operatorname{cat}_{L}\left([f]_{R}\right):=\operatorname{cat}(f)$.

3. If $[g] \leqq_{L R}[f]$, i.e., $g \sim h \circ f \circ s$ with $s: X \rightarrow X$ and $t: Y \rightarrow Y$, then we have

$$
\operatorname{cat}(g)=\operatorname{cat}(t \circ f \circ s) \leqslant \min \{\operatorname{cat}(t), \operatorname{cat}(f), \operatorname{cat}(s)\} \leqq \operatorname{cat}(f) .
$$

Hence we have $\operatorname{cat}(g) \leqq \operatorname{cat}(f)$. Thus $\operatorname{cat}_{L R}:[X, Y]_{L R} \rightarrow\left(\mathbb{Z}_{\geqq 0}, \leqq\right)$ is a poset map. Here $\operatorname{cat}_{L R}\left([f]_{R}\right):=\operatorname{cat}(f)$. 
Namely we have the following commutative diagrams:
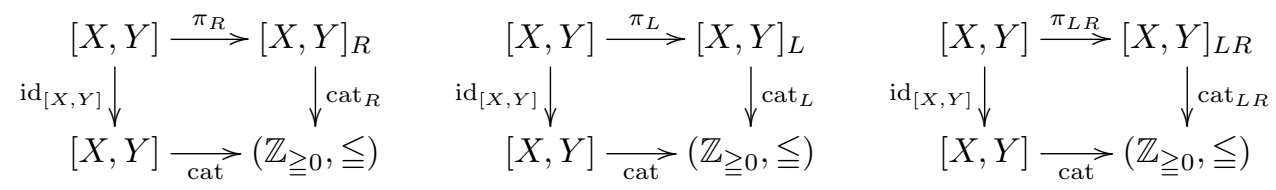

Remark 4.23. Finally, we remark that the referee pointed out that our machinery might be relevant to, for example, the following examples:

1. The theorem of Dehornoy $[10,11,12]$ about natural orders on braid groups (e.g., see [14]), which has given rise to considerable activity in low-dimensional topology, such as generalizations to knot group.

2. Elmendorf's theorem in equivariant homotopy theory, which describes $G$-equivariant homotopy types in terms of fixed-point spaces indexed by the orbit category of homogeneous spaces $G / H$ and $G$-maps between them (e.g., see [24]): this yields natural stratifications of $G$-spaces.

3. Some related connections between homotopy theory and (equivariant) posets, e.g., such as a theorem saying that the category of $(G-)$ posets admits a model structure that is Quillen equivalent to the standard model structure on the category of topological $(G-)$ spaces $^{3}$ (e.g., see $[28,32,37]$ ).

Furthermore the referee pointed out that he/she suspects that in the long run such poset structures will find an interpretation as part of Connes-Consani's recent theory "Homological algebra in characteristic one" [8].

In this paper we deal with only the homotopy set $[X, Y]$. However, if other things, e.g., the above examples and Connes-Consani's recent theory, are relevant to our machinery, then it would be quite interesting.

\section{Acknowledgments}

We would like to thank Jim Stasheff for informing us of papers by K. Borsuk and P. Hilton, and also the referee for his/her pointing out those interesting/intriguing examples or works and Connes-Consani's recent theory.

\section{References}

[1] P. Alexandroff, Diskrete Räume, Mat. Sb. (N.S.) (1937), 501-518.

[2] P. Alexandroff, Combinatorial Topology, Dover Publications, Inc., 31 East 2nd Street, Mineola, NY, 1947.

[3] F. Arenas, Alexandroff spaces, Acta Math. Univ. Comenian. LXVIII, No. 1 (1999), 17-25.

[4] D. Ayala, J. Francis and H.L. Tanaka, Local structures on stratified spaces, Adv. Math. 307 (2017), 903-1028.

\footnotetext{
${ }^{3}$ In [28, page 83] they write "This implies that all of the algebraic topology of spaces can in principle be worked out in the category of posets. It can also be viewed as a bridge between the combinatorics of partial orders and algebraic topology."
} 
[5] J.A. Barmark, Algebraic Topology of Finite Topological Spaces and Applications, Lecture Notes in Math., vol. 2032, 2011.

[6] K. Borsuk, Sur la notion de diviseur et de multiple des transformations, Bull. Acad. Pol. Sci. 3 (1955), 81-85.

[7] K. Borsuk, Sur la notion de dépendence des transformations continues, Bull. Acad. Pol. Sci. 3 (1955), 251-254.

[8] A. Connes and C. Consani, Homological algebra in characteristic one, arXiv: 1703.02325v1[math AG].

[9] J.M. Curry, Sheaves, cosheaves and applications, University of Pennsylvania, Ph.D Thesis, 2014, arXiv: 1303.3255v2.

[10] P. Dehornoy, Braid groups and left distributive operations, Trans. Amer. Math. Soc. 345 (1994), 115-150.

[11] P. Dehornoy, From large cardinals to braids via distributive algebra, J. Knot Theory Ramifications 4 (1995), 33-79.

[12] P. Dehornoy, A fast method for comparing braids, Adv. Math. 125 (1997), 200-235.

[13] Y. Félix, S. Halperin and J.C. Thomas, Rational Homotopy Theory, Grad. Texts in Math., vol. 205, Springer-Verlag, 2001.

[14] R. Fenn, M.T. Greene, D. Rolfsen, C. Rourke and B. Wiest, Ordering the braid groups, Pacific J. Math. 191, No. 1 (1999), 49-74.

[15] D. Gottlieb, A certain subgroup of the fundamental group, Amer. J. Math. 87 (1965), 840-856.

[16] D. Gottlieb, Evaluation subgroups of homotopy groups, Amer. J. Math. 91 (1969), 729-756.

[17] A. Hatcher, Algebraic Topology, Cambridge University Press, Cambridge, 2001.

[18] P. Hilton, On divisors and multiples of continuous mappings, Fund. Math. 43 (1957), 358-386.

[19] P. Hilton, On some contributions of Karol Borsuk to homotopy theory, Topol. Methods Nonlinear Anal. (J. Juliuss Schauder Center) 1 (1993), 9-14.

[20] P. Hilton, Duality in homotopy theory: a retrospective essay, J. Pure Appl. Algebra 19 (1980), 159-169.

[21] P. Hilton, G. Mislin and J. Roitberg, Localization of Nilpotent Groups and Spaces, North-Holland Math. Stud., vol. 15, 1975.

[22] S.-T. Hu, Homotopy Theory, Academic Press, 1959.

[23] J. Lurie, Higher Algebra, version of March 2016, available at http://www . math . harvard.edu/ lurie/papers/HA.pdf.

[24] J.P. May, Equivariant Homotopy and Cohomology Theory: Dedicated to the Memory of Robert J. Piacenza, with contributions by M. Cole, G. Comezaña, S. Costenoble, A.D. Elmendorf, J.P.C. Greenlees, L.G. Lewis, Jr., R.J. Piacenza, G. Triantatillou, and S. Waner, CBMS Regional Conference Series in Mathematics, No. 91, 1993, DOI: http://dx.doi.org/10.1090/cbms/091.

[25] J.P. May, Finite topological spaces. Notes for REU (2003), http://www.math. uchicago.edu/ may/MISCMaster.html. 
[26] J.P. May, Finite spaces and simplicial complexes. Notes for REU (2003), http: //www. math.uchicago. edu/ may/MISCMaster.html.

[27] J.P. May, Finite groups and finite spaces. Notes for REU (2003), http://www . math.uchicago.edu/ may/MISCMaster.html.

[28] J.P. May, I. Zakharevich and M. Stephan, The homotopy theory of equivariant posets, Cah. Topol. Géom. Différ. Catég. 58, No. 2 (2017), 82-114.

[29] M.C. McCord, Singular homology and homotopy groups of finite spaces, Notices Amer. Math. Soc. 12 (1965), 622.

[30] M.C. McCord, Singular homology groups and homotopy groups of finite topological spaces, Duke Math. J. 33 (1966), 465-474.

[31] J. Milnor and J. Stasheff, Characteristic Classes, Ann. of Math. Stud., Princeton Univ. Press, 1974.

[32] G. Raptis, Homotopy theory of posets, Homology Homotopy Appl. 12, No. 2 (2010), 211-230.

[33] T. Speer, A short study of Alexandroff spaces, arXiv:0708.2136.

[34] R.E. Stong, Finite topological spaces, Trans. Amer. Math. Soc. 123 (1966), $325-340$.

[35] D. Tamaki, Cellular stratified spaces, in "Combinatorial and Toric Homotopy", Lecture Notes Series, Institute for Mathematical Sciences, National University of Singapore, vol. 35, World Scientific, 2017, pp. 305-435.

[36] R. Thom, Opérations en cohomologie réele, Séminaire Henri Cartan, Paris $1954 / 5$.

[37] R.W. Thomason, Cat as a closed model category, Cah. Topol. Géom. Différ. Catég. 21, No. 3 (1980), 305-324.

[38] M.H. Woo and K.Y. Lee, The relative evaluation subgroup of $C W$-pair, J. Korean Math. Soc. 25 (1988), 149-160.

[39] J. Woolf, The fundamental category of a stratified space, J. Homotopy Relat. Struct. 4 (2009), 359-387.

[40] T. Yamaguchi and S. Yokura, A proset structure induced from homotopy classes of maps and a classification of fibrations, To appear in Commun. Korean Math. Soc.

[41] S. Yokura, Decomposition spaces and poset-stratified spaces, preprint, 2017.

[42] Y.S. Yoon, The generalized dual Gottlieb sets, Topology Appl. 109 (2001), $173-181$.

Toshihiro Yamaguchi tyamag@kochi-u.ac.jp

Faculty of Education, Kochi University, 2-5-1, Kochi, 780-8520, Japan

Shoji Yokura yokura@sci.kagoshima-u.ac.jp

Department of Mathematics and Computer Science, Graduate School of Science and Engineering, Kagoshima University, 1-21-35 Korimoto, Kagoshima, 890-0065, Japan 